\title{
¿Cómo Surge el Lenguaje en el Niño?. Los planteamientos de Piaget, Vygotski y Maturana
}

Nolfalbáñez S. ${ }^{1}$

\begin{abstract}
Resumen
El trabajo contrasta el planteamiento de Humberto Maturana con los aspectos básicos de las teorías de Jean Piaget y L:S: Vygotski sobre el surgimiento del lenguaje. Se discuten las conceptualizaciones propias de cada teoria con respecto a los fundamentos, factores o circunstancias con los que los autores explican la constitución del lenguaje en el niño. El propósito de esta contrastación es contribuir a la comprensión de la nueva visión sobre el lenguaje como generador de realidades que aporta la biología del conocimiento de H. Maturana, y que permite cambiar la mirada sobre las condiciones de constitución en el niño del fenómeno que nos hace propiamente humanos, posibilitando una proyección distinta e innovadora al trabajo pedagógico y psicopedagógico en el área del lenguaje y la comunicación.
\end{abstract}

\section{Abstract}

This paper confronts the theory of Humberto. Maturana on the appearance of language in the early childhood with those of Jean Piaget and L.S. Vygotski. The central discussion is about the foundations, elements and circumstances that each author considers in his explanation of the first emergence of language in the individual. The purpose of this comparison is to make a contribution to the understanding of the new perspective on language present in Maturana's theory, which constitutes a new vision of this phenomenon and allows a different and innovative approach to the psycopedagogic work in the field of language and communication.

Key words: Languaje, Child, reality.

Las corrientes teóricas explicativas del conocimiento y del aprendizaje que tienen mayor influencia en el ámbito educacional por ser consideradas fundamentos de la perspectiva constructivista que hoy se propicia, provienen de la psicologia: la epistemologia genética de Jean Piaget y la teoria del desarrollo social de los procesos cognitivos de Lev S. Vygotski. Ambos autores, en el marco de sus respectivas teorias del conocimiento, han desarrollado una explicación sobre el surgimiento del lenguaje en el niño, cada uno desde diferentes perspectivas y con distintos énfasis, pero coincidiendo en la concepción de lenguaje como un instrumento de la co- municación cuya aparición es independiente del pensamiento.

En Chile, el biólogo y epistemólogo Humberto Maturana R., considerado un constructivista radical, plantea el fenómeno lingüístico como el resultado de una historia de interacciones recurrentes posibilitada por un espacio relacional donde lo central es la disposición emocional de aceptación del otro como un legítimo otro. En su teoria cognoscitiva, la biología del conocimiento, Maturana desarrolla una proposición explicativa sobre las condiciones de generación del lenguaje, el que se constituiria cuando los participantes en la

1 Prof. Departamento de Educación Diferencial, Facultad de Filosofiay Educación, Univ. Metropolitanade Ciencias de la Educación. 
interacción operan en una clase especial de acciones -coordinaciones de acciones consensuales de coordinaciones de acciones consensuales- en la cual surge el observador y con él, el mundo de objetos y relaciones. En esta perspectiva no hay separación ni independencia entre lenguaje y pensamiento, por lo que uno no puede ser instrumento del otro.

El presente trabajo pretende contrastar el planteamiento de Maturana con los aspectos básicos de las teoria de Piaget y de Vygotski sobre el lenguaje y sobre los fundamentos, factores o circunstancias con los que ellos explican su constitución en el niño. Ésto, con el propósito de contribuir a la comprensión de la nueva visión sobre el lenguaje como generador de realidades que aporta la biología del conocimiento y que permite cambiar la mirada sobre el fenómeno que nos hace propiamente humanos, posibilitando una proyección distinta e innovadora al trabajo pedagógico y psicopedagógico en el área del lenguaje y la comunicación.

\section{Elplanteamientode JeanPiaget}

Según Piaget, toda adquisición cognoscitiva incluido el lenguaje, es una construcción progresiva a partir de formas evolutivas de la embriogénesis biológica hasta el pensamiento científico contemporáneo. Entiende el lenguaje como lenguaje articulado, socialmente transmitido por educación y asimila su constitución a la aparición del lenguaje verbal. Para él lenguaje propiamente dicho es “... palabras, frases elementales, luego sustantivos y verbos diferenciados, y por último frases completas.(Piaget, 1983:34). El lenguaje sería un caso particular de la función semiótica o simbólica que se constituye en el curso del segundo año y que en su conjunto (imitación diferida y simbólica de los gestos, juego simbólico, imagen mental, imagen gráfica o dibujo, etc.) es la responsable del paso desde las conductas sensorio-motrices hasta el nivel de representación o pensamiento, y no el lenguaje solo (Piaget, J.; Inhelder, B; 1982; Piaget, J.; Chomsky, N.; 1983).
Para Piaget, la función simbólica se establece con la representación previa al lenguaje que nace de la unión de "significantes" que permiten evocar objetos ausentes relacionándolos con los elementos presentes. Esta conexión entre significante y significado es lo característico de la función simbólica, “... el simbolo se basa en el simple parecido entre el objeto presente que juega el papel de "significante" y el objeto ausente "significado", lo cual implica una representación: una situación no dada es evocada mentalmente y no sólo anticipada como un todo en función de alguna de sus partes" (Piaget, 1982: 137) Piaget sostiene que antes del lenguaje hay una inteligencia pero no hay pensamiento, distingue la inteligencia como la coordinación de medios para lograr un objetivo que no es accesible de inmediato y que se daria en un proceso de desarrollo equiparable con el desarrollo orgánico y dependiente de él. El pensamiento es para él la inteligencia interiorizada que no se apoya sobre la acción directa sino que sobre un simbolismo. La característica fundamental de la inteligencia previa al lenguaje seria la búsqueda de un fin, la solución de un problema práctico; en suma, la intencionalidad de los actos del niño pequeño. En las observaciones realizadas por Piaget con sus hijos para el estudio de la imitación, registró las conductas de imitación del bebé como "respuestas" de éste a los modelos sonoros emitidos por el adulto, asignando a esas respuestas un carácter intencionado. (Piaget, J.; 1980, 1983). Como conclusión de estos estudios, afirma que la génesis de la imitación en los dos primeros años "no tiene nada de automática o de involuntaria ( en el sentido de no intencional); por el contrario, demuestra muy pronto coordinaciones inteligentes, tanto en el aprendizaje de los medios que emplea cuanto en sus fines mismos" (Piaget, 1982 :17).

En la perspectiva piagetiana, el lenguaje no es suficiente para explicar el pensamiento, no es condición suficiente, porque las estructuras que lo caracterizan tendrian sus raices en la acción y los mecanismos sensorio motores propios de estas estructuras serian más profundos que los lingüisticos. La fuente del pensamiento sería la función simbólica que engloba al sistema de signos verbales y a 
todo sistema de símbolos, y el factor que aseguraría la transición entre las conductas sensorio motrices y las conductas simbólicas o representativas sería la imitación. Para Piaget, la imitación tiene un papel preponderante en la constitución de la función simbólica que incluye al lenguaje: "... la imitación parece constituir un mecanismo esencial en la formación de esta función semiótica o simbólica, en tanto que la imitación sensorio-motriz constituye ya una suerte de representación pero en actos" (Piaget, J.; 1981: 45). “...donde se adquiere el lenguaje es sobre todo en un contexto de imitación, y este factor imitativo parece constituir un auxiliar esencial, porque, si el aprendizaje del lenguaje sólo fuera debido a condicionamientos, debería ser mucho más precoz". (Piaget, J.; 1980:136). Piaget reconoce como posible objeción a su teoria sobre el rol de la imitación, el hecho que los niños observados pudieran atribuir a los sonidos espontáneos y diversos que se les hacian alguna significación global inherente a esa repetición, pero entonces, dice él, "toda producción vocal estaría en el mismo caso y no se podría caracterizar la imitación por el deseo de reproducir los sonidos significativos con exclusión de los otros" (Piaget, J.; 1982 :39). Es evidente que el autor no concibe siquiera la posibilidad de que esta imitación de sonidos no sea intencionada.

Piaget hace una distinción entre símbolos y signos, siendo estos últimos los únicos a los que atribuye un sentido social, puesto que el simbolo que es la imagen previa al lenguaje la considera estrictamente individual: “... la imitación representativa precede a la imagen y no la sigue, y asi el simbolo interior es un producto de interiorización y no un factor nuevo que surge de no se sabe dónde (...) La imagen mental sigue siendo de orden privado (...) y no sirve sino para traducir sus experiencias privadas” (Piaget; op. cit.: 97-98); “... el signo supone una relación social - como es claro en el lenguaje o en el sistema de signos verbales- en tanto que la motivación (o parecido entre el significante y el significado) característica del símbolo, podría ser el producto del pensamiento simplemente individual" (Piaget; 1981:137-138). Aquí, el pensamiento partiría de cero en cuanto contenido conceptual, pero no en cuanto datos sensorio-motrices y perceptivos. Además, el pensamiento tendría una preparación funcional de la cual serian respnsables tanto las coordinaciones sensorio-motoras y nerviosas como lo que el funcionamiento nervioso ha heredado del funcionamiento orgánico en general.

Primero el símbolo sería la imagen, la representación no comunicable, privada, inherente a los mecanismos individuales del pensamiento y por lo tanto producto de interiorización; después surgiría el lenguaje como parte de la función simbólica y como caso separado de la representación de alguna cosa por medio de otra cosa, de la imitación diferida (simbolismo gestual) y de la imitación interiorizada o imagen mental. Todos aparecerian alrededor de los 18 meses y harian posible el pensamiento. Piaget no considera al lenguaje como un aspecto central en el desarrollo cognoscitivo del niño sino más bien como un producto de él, y afirma que "el lenguaje no transforma el pensamiento sino en la medida en que éste se encuentra apto para dejarse transformar" (Piaget, J.; 1982: 11). Para él, las relaciones del niño con otros, previas a la adquisición del lenguaje propiamente dicho, se limitan a la imitación de gestos corporales y a una relación afectiva global sin comunicaciones diferenciadas (Piaget, J. 1983). Lo social no sería un factor importante en la aparición de las conductas propias de la etapa sensorio motriz ya que éstas serían producto de la acción individual propia de ese estadio del desarro1lo: "Puede observarse, pues, ya mucho antes del lenguaje, un juego de las funciones sensoriomotrices que es un juego de puro ejercicio, sin intervención del pensamiento ni de la vida social, ya que no pone en acción más que movimientos y percepciones." (Piaget, J. 1983:33, la cursiva es mía). De este modo, para Piaget el símbolo no es un producto social sino un producto de la inteligencia sensorio motriz, no cree que el símbolo se deba concebir como un producto social debido al intercambio y a la comunicación intelectual, y su primer argumento es que "no siempre la palabra o el contacto con otro acompañan a la formación de un simbolismo" (Piaget; 1982: 139). 
El lenguaje para este autor es, al igual que toda adquisición cognoscitiva, una construcción progresiva que se sustenta en la inteligencia que es previa; y la conducta lingüística, al igual que toda conducta, es un producto de los procesos de asimilación y acomodación con los que él explica el conocimiento: “... toda conducta es una asimilación de lo dado a esquemas anteriores y toda conducta es al mismo tiempo acomodación de estos esquemas a la situación actual (...) toda conducta tiende a asegurar un equilibrio entre los factores internos y externos o, más generalmente, entre la asimilación y la acomodación" (Piaget, J., 1983: 147). El autor postula que tanto el crecimiento físico como el intelectual tienen sus "creodas" (rutas necesarias), y que la actividad cognoscitiva supone acercarse a lo real; es más , afirma que "el conocimiento tiene como función esencial captar el medio" (Piaget, 1981: 23), pero que el organismo sólo capta los estímulos hacia los que está sensibilizado, es decir, aquellos que asimila activamente a esquemas previos de respuesta. Los estadios que Piaget describe en el desarrollo intelectual del niño no están predeterminados sino son una construcción progresiva, pero reconoce una dirección en estos estadios que él concibe como una tendencia al equilibrio o a un estado de perfeccionamiento en el desarrollo; en sus palabras: "todo desarrollo culmina con un estado de equilibrio relativo, e inclusive tiende a él en virtud de sus mecanismos autorreguladores" (Piaget, 1981: 78). Piaget no establece cómo es que los procesos cognitivos en desarrollo se involucran o intervienen en el desarrollo lingüístico. Al respecto, J. Bruner dice: "El lenguaje aparece como un sintoma de la semiotización automática de las operaciones cognitivas del desarrollo, y no se especifica por qué medios concretos estas operaciones cognitivas no lingüísticas propician el nacimiento de la capacidad para reconocer y emplear la gramática de predicados, o del sistema de marcadores ling. definido-indefinido de la anáfora, o la capacidad de generar únicamente frases bien formadas, confiando asi, con una fe ciega, en la inevitabilidad del progreso" (Bruner, J. 1989: 174).
Si bien Piaget reconoce el papel esencial de lo social en la elaboración conceptual y en la aparición de los esquemas representativos ligados al lenguaje, lo social no explicaría por sí solo el comienzo de la imagen o del simbolo, ya que él establece este comienzo en la imitación o en los juegos imaginativos de los niños al año de edad como producto de interiorización individual. La existencia previa de la función simbólica es lo que haria posible las interacciones "pensadas" o conscientes entre individuos y la posterior constitución de significaciones colectivas por medio de signos sociales. Para este autor, el surgimiento del lenguaje es independiente del desarrollo de la inteligencia sensorio-motriz y posterior a ella, por lo que no tendría intervención alguna en los esquemas de comportamiento anteriores a la constitución de la función simbólica. Los esquemas operatorios descritos por el autor como propios del desarrollo cognoscitivo del niño, derivarian directamente de los esquemas de acción y las estructuras lógico.matemáticas provendrian de la coordinación de las acciones que el niño realiza mucho antes de utilizar lenguaje; por ejemplo, la operación de suma derivaria de la acción de reunir del niño pequeño, acción en la cual ni el lenguaje ni lo social intervendrian.

En esta explicación, la acción del niño se hace social con el lenguaje, es decir, lo social surge con el intercambio interindividual que es producto de lo que élllama "lenguaje propiamente dicho". Sólo a partir de la adquisición del lenguaje mediante los procesos de asimilación y acomodación y con la intervención de la imitacion, se haria posible el intercambio interindividual y el niño se veria enfrentado al mundo social. Este sería recién el inicio de la socialización de la acción.

\section{ElenfoquedeMaturana}

En la perspectiva de la biologia del conocimiento, el lenguaje es el operar en coordinaciones de acciones consensuales de coordinaciones de acciones consensuales. Maturana propone un mecanis- 
mo generativo del cual resulta el lenguaje entendido de este modo y que puede ser presentado esquemáticamente como sigue:

- En el espacio relacional posibilitado por un fenómeno biológico: una disposición inicial de aceptación, una emoción que Maturana llama amor y que significa la aceptación del otro junto a uno, se hace posible la coordinación de las acciones (coordinación conductual) de los participantes en la interacción.

- Si la emoción de aceptación permanece y los participantes en la interacción no se separan, se da la recurrencia de esas coordinaciones de acciones, permitiendo que se expandan y que puedan hacerse consensuales; es decir, aprendidas, de mutuo entendimiento práctico, en el sentido de que las conductas nuevas que surgen en esa relación son correspondientes a esa historia de interacciones y uno entiende que no podrian haber surgido con independencia de ella.

- El lenguaje se constituye cuando el observador puede describir interacciones en las cuales los participantes que operan en coordinaciones de acciones consensuales aplican las consecuencias de su operar anterior en coordinaciones de acciones consensuales a su interacción presente; es decir, el lenguaje surge cuando en el fluir de sus interacciones recurrentes esas coordinaciones de acciones consensuales se hacen recursivas; cuando el observador puede distinguir coordinaciones de acciones consensuales de coordinaciones de acciones consensuales.

En relación a la noción de desarrollo, para Maturana ésta surge en el contexto de la observación, que es un dominio distinto al del operar del organismo. La ontogenia para Maturana, en cuanto proceso, no representa el paso de un estado incompleto a otro más completo o definitivo, "sino la manifestación de un sistema que es en cada instante la unidad en su totalidad" (Maturana, H. y Varela, F; 1995:77). En este mismo sentido, y a diferencia de Piaget, Maturana sostiene que "la aproximación a un estado final que se observa en el desarrollo de una conducta es una consecuencia histórica de un proceso de deriva, y no una transformación activa (Maturana, H. 1993:223, la cursiva es mía). Si la finalidad se entiende como un factor presente en la realización misma de la conducta, entonces el estado final tendría que estar de alguna manera siempre actuando en dicha realización; para ello, dice Maturana, "... como científico, requeriría un mecanismo, y ese mecanismo no está. En la medida que el organismo es un sistema determinado estructuralmente ese mecanismo no está, y no hay nada en los procesos de desarrollo en los cuales pueda uno decir que el estado final está presente como factor en su realización. No hay mecanismos para eso" (op.cit.:235).

Para Piaget, incluso la imitación vocal que el bebé hace antes de los seis meses es una actividad intencionada. Por su parte, Maturana afirma que la intención surge en la descripción del observador en el lenguaje de lo que ocurre en el lenguaje: "Intención es una disposición a una acción con ciertas consecuencias, en la cual la visión de las consecuencias es parte del cómo se está en la acción ahora. Si uno no puede ver (mostrar) esa relación, no puede hablar de intención" (Maturana, H. 1991 ). Maturana define la intención como una reflexión del observador, como una relación que establece el observador entre un estado inicial y uno final, pero no la admite como una explicación científica causal de la actividad humana, puesto que existe determinación estructural y no pueden haber mecanismos generativos que expliquen cómo en el operar del ser humano está presente el estado final como factor de la realización de la actividad presente. Considerar la intención como fundamento del lenguaje significaria que el lenguaje surge desde la intencionalidad del niño pequeño, lo que requeriria dar cuenta de la operacionalidad que constituye laintención previa al lenguaje, puesto que el orientar la conducta hacia sus consecuencias, que es lo que define las intenciones, presupone estar ya en el lenguaje . Dicho de otro modo, afirmar que la intención fundamenta el fenómeno del lenguaje implica que la intención es previa a su 
surgimiento, por lo que el niño debería tener, de algún modo, la "intención de" como requisito para operar en el lenguaje. Esto significaria que los fines o metas hacia los cuales se orienta la conducta existirian ya para el niño de manera parecida a como existen para el adulto, lo que no es posible fuera del lenguaje (Ibáñez, N. 1998). La atribución de intenciones a la conducta del niño las hace el observador como si el niño compartiera con él un mundo de objetos y relaciones preexistente, porque no considera la posibilidad de que ese mundo sólo puede surgir en el lenguaje. En la perspectiva de la biología del conocimiento, la intención no es un rasgo central del lenguaje sino un comentario del observador acerca del fluir de las coordinaciones consensuales recursivas de acciones que constituyen el lenguaje, “... los conceptos como intencionalidad adquieren su propia pertinencia como consecuencia del flujo del lenguajear, y no pueden confundirse con sus fundamentos" (Maturana, H. 1997:40).

En cuanto a la explicación de Piaget sobre cómo se logran las adquisiciones cognoscitivas entre las que se encuentra el lenguaje, Maturana piensa que, indirectamente, Piaget valida la captación de una realidad externa como parte del mecanismo de sobrevida del organismo: “...él (Piaget) en la noción de asimilación, no es claro sobre en qué medida acepta el determinismo estructural del organismo como una imposibilidad de captar un mundo externo. Mi impresión es que no lo acepta de esa manera, y que él visualiza los fenómenos de asimilación y acomodación como procesos en los cuales los organismos están orientados a captar realidades ambientales, y a acercarse a ellas en el proceso del vivir. Yo pienso distinto" (Maturana, H. 1993:223).

Por una parte, la noción de asimilación de algo externo aparece contradictoria con el planteamiento de Maturana respecto a la no interacción entre los dominios de existencia de los seres humanos, el de la neurofisiología y el de la conducta; puesto que para Piaget existe datos sensorio-motrices como antecedentes del pensamiento que preparan funcionalmente las adquisiciones cognoscitivas, entre las cuales está el lenguaje, del mismo modo que lo hace lo que se ha heredado del funcionamiento orgánico y nervioso general. Para Maturana, la neurofisiología y la conducta son dominios disjuntos que nunca se intersectan, aún cuando tienen una relación generativa producto de la mutua contingencia en el curso de sus respectivos cambios; el lenguaje es un fenómeno biológico porque resulta del operar de seres humanos, pero el lenguaje es una dinámica relacional que no se da en el cuerpo de los participantes sino en el fluir de la relación; el lenguaje "ocurre en el dominio de las coordinaciones de acciones de los participantes y no en su fisiología o neurofisiología (...) el lenguaje como un tipo especial de operación en coordinaciones de acciones, requiere de la neurofisiología de los participantes, pero no es un fenómeno neurofisiológico" (Maturana, H. 1997: 50). Por otra parte, la afirmación de Piaget respecto a que el conocimiento tiene como principal función captar la realidad, acercarse a lo real, implicaria que él no considera la determinación estructural del ser vivo, por lo que la construcción progresiva que define como una autorregulación en sí misma tendería a ser construcción de algo lo más parecido posible a lo real, al mundo de objetos físicos o conceptuales que existirian con independencia del observador. Sin embargo, Piaget sostiene al mismo tiempo que el organismo sólo capta aquellos estímulos hacia los cuales está sensibilizado en determinados momentos del desarrollo, aquellos que puede asimilar activamente a esquemas previos, lo que parece implicar la idea de que lo externo no especifica lo que le pasa al organismo, o, al menos, que lo externo no siempre especifica lo que le pasa al organismo.

Para Maturana, la imposibilidad que tiene el organismo de captar estímulos del medio es un aspecto central de su teoría, en la cual el autor se hace cargo de la determinación estructural del ser vivo que no puede distinguir entre ilusión y percepción en la experienciay, en consecuencia, no puede tener acceso a una realidad independiente. Según Maturana, lo que le pasa a un ser vivo está siempre determinado en su estructura y no por el agente perturbante, por lo que el medio no puede ser ins- 
tructivo. Su proposición explicativa la desarrolla en lo que él llama "el camino de la objetividad en paréntesis", como un camino explicativo distinto al "objetivo" que supone que se puede tener acceso a un mundo independiente de lo que hace el observador. Al respecto, Maturana sostiene: “...los observadores que toman el camino explicativo de la objetividad sin paréntesis no pueden evadir tomar el lenguaje como un sistema de comportamiento que ellos usan para comunicarse entre ellos respecto de entidades que existen independientemente de lo que hacen. Aun más, haciendo esto ellos no pueden evadir el supuesto implícito de que ellos tienen la habilidad constitutiva para captar la existencia y las caracteristicas de tales entidades independientes, y de simbolizar tanto su existencia como sus características con palabras. 0 sea, en este camino explicativo el observador que quiera hablar sobre el lenguaje no puede evadir hablar sobre palabras como si éstas fueran símbolos que soportan entidades independientes sobre las cuales ellos se comunican entre si." (Maturana, H. 1997: 48). Para Maturana el símbolo es secundario al lenguaje, porque para operar con simbolos se requiere estar ya en el lenguaje. El símbolo es una reflexión que un observador hace sobre relaciones que distingue en el curso de su operar en el lenguaje: "Los símbolos surgen ... en la recursión de las coordinaciones de coordinaciones conductuales como distinciones no intencionales de clases de objetos (entidades) fuera de la concretitud inmediata del hacer, pero que connotan ese quehacer" (Maturana, $\mathrm{H}$. 1997:130).

En relación a lo social, para la biología del conocimiento siempre que hay seres humanos que interactúan recurrentemente en un espacio de aceptación, hay un hecho social. Según Piaget, las coordinaciones sensorio-motoras y la función simbólica previa al lenguaje son mecanismos individuales y no sociales; ¿significa ésto que aún cuando el bebé no esté en interacción con otros igualmente la función simbólica apareceria a la edad aproximada que establece Piaget?. Siaceptamos con Maturana que la conducta es una relación entre el individuo y su medio que un observador describe, ¿cómo podría una conducta de imitación que prepara o antecede la adquisición del lenguaje, ser individual y no social?; esto no parece posible, si la conducta es siempre relacional no podria el bebé interactuar recurrentemente con otra persona en un sentido no social, con independencia si quien realiza la conducta está en el lenguaje o no, puesto que quien distingue la realización de tal conducta es siempre un observador (en el lenguaje).

\section{Elplanteamientode L. S. Vygotski}

Vygotski, a diferencia de Piaget, sostiene que el lenguaje es primario con respecto al desarrollo intelectual del niño. El lenguaje es previo, por lo que este desarrollo va de lo social a lo individual, y se establece en el proceso social que lo posibilita: "el pensamiento no se expresa simplemente en palabras sino que existe a través de ellas." (Vygotski, L.; 1988:166); ".. el desarrollo del pensamiento está determinado por el lenguaje, es decir, por las herramientas lingüísticas del pensamiento y la experiencia socio-cultural del niño (...) El crecimiento intelectual del niño depende del dominio de los medios sociales del pensamiento, esto es, del lenguaje" (op.cit.: 80). En esta perspectiva, lo esencial para la constitución del lenguaje es lo social: "La transición desde el mundo animal hasta el nivel humano significa la aparición de un nuevo principio de desarrollo (...), con la transición al hombre, la forma básica del desarrollo mental pasa a ser la adquisición de las experiencias de otros mediante la práctica conjunta y el lenguaje" (Luria y Yudovich; 1979: 11). Vygotski difiere de Piaget respecto al carácter fundamental del lenguaje con respecto al desarrollo intelectual, pero coincide con él en considerar que el desarrollo ontogenético del lenguaje y del pensamiento tienen distintas raíces genéticas y cursan en lineas separadas.

Vygotski distingue una etapa preintelectual en el desarrollo del lenguaje y una etapa prelingüística en el desarrollo intelectual, estas lineas de desarrollo serían independientes hasta un cierto punto y 
luego se juntarian: “... en un momento determinado estas lineas se encuentran y entonces el pensamiento se torna verbal y el lenguaje racional." (Vygotski; 1988:72). En la perspectiva de este autor, el lenguaje surge cuando el niño se refiere al objeto de la realidad, y esta referencia a lo objetivo es la caracteristica del signo (simbolo-palabra) que es un estimulo artificial creado por el hombre como medio para dominar la conducta propia o ajena y que supone la generalización y el significado. Aquí haabría lenguaje cuando la palabra representa al objeto en la conciencia, lo que ocurriria gradualmente dado que durante los primeros años la palabra sería más una propiedad del objeto que un simbolo de éste, y sólo en el proceso de operar con ellas el niño descubriría y consolidaría la función de esas primeras palabras como signos (Vygotski, L.; 1988).

Según Vygotski, el prelenguaje es la fase del desarrollo del lenguaje que corresponde a las primeras palabras (emisiones) del niño que aún no están diferenciadas del contexto situacional y de la acción. Esta fase sería independiente del desarrollo del pensamiento y duraria hasta el año y medio, período que define como de transición de la vida natural a la cultural. Para él, sólo hasta los nueve meses el comportamiento del niño estaría supeditado a las posibilidades estructurales de sus órganos al igual que en otros animales, después de esa edad se produciria un viraje y lo cultural significaría la ampliación de esas primeras posibilidades. El primer año y medio correspondería a lo que él llama la etapa prehistórica en relación al lenguaje, donde las conductas prelingüísticas del niño no serian diferentes a las de otros animales no humanos, lo que para Vygotski explicaría el que no exista recuerdo del primer año de vida; en este período no habría intención, las primeras emisiones verbales del niño no serían acción intencionada. Al respecto, Vygotski cita la opinión que se remonta a Spinoza: "elalma no puede cumplir ninguna intención si no la recuerda" (Vygotski,L.; 1995: 299). Hasta el año y medio la palabra tendria la función de indicar, que sería la función primaria en el desarrollo del lenguaje; las primeras palabras iniciarian la sustitución de los gestos indicadores previos por signos convencionales, y esas primeras palabras harían referencia a toda una situación incluyendo la acción del niño que las emite; es decir, estarian aún ligadas a la acción. A diferencia de Piaget, Vygotski critica la atribución de intenciones a la actividad del niño durante el primer año y medio, puesto que para él la actividad dirigida a un fin es propia de etapas posteriores y el supuesto de que toda acción humana es intencional impide o priva de la posibilidad de determinar cuándo se dan por primera vez acciones dirigidas a un fin en el niño pequeño. Para él, "la intención constituye un proceso típico de dominio de la propia conducta mediante la creación de las siguientes conexiones correspondientes, (..) La intencionalidad se basa precisamente en crear una acción que se deduce de la exigencia directa de las cosas (op.cit.:291). Vygotski reconoce dos tendencias como raíces del lenguaje por su proceso evolutivo, la tendencia expresiva y la comunicativa que devienen en las respectivas funciones, y no admite que exista una tendencia intencional, puesto que no habría para ella un proceso evolutivo ni factores condicionantes: “... la tendencia intencional surge de la nada, carece de historia, nada la condiciona" (Vygotsky; 1995a: 85).

Para Vygotski, el que sea evidente que en el niño pequeño no hay actividad intencionada comprueba su tesis sobre las lineas de desarrollo ontogenético independientes del pensamiento y del lenguaje, puesto que la intención sería propia del pensamiento: "Es casi imposible atribuir a un niño de año y medio una conciencia o pensamiento plenamente formado. Cuando el niño grita, lo que menos puede suponerse es que sabe por experiencia lo que va a suceder entre el grito y las acciones sucesivas de la gente que lo rodea o que su grito pueda compararse con nuestras intencionadas acciones o comunicaciones cuando hablamos para influir sobre la gente. Es indudable, por lo tanto, que la primera fase del desarrollo del lenguaje infantil no está relacionada en absoluto con el desarrollo del pensamiento infantil; no está relacionada con el desarrollo de los procesos intelectuales del niño. (...) el desarrollo del lenguaje transcurre al principio independientemente del desarrollo del 
pensamiento, con la particularidad de que en sus primeras etapas se desarrolla de un modo más o menos igual tanto en los niños normales como en los profundamente atrasados." (Vygotski, L.; 1995:171-172). De este modo, Vygotski descarta la intencionalidad en la actividad del niño pequeño, a diferencia de Piaget y de las perspectivas lingüísticas, y postula que el sentido del lenguaje surge en el desarrollo de la intencionalidad, que "la orientación hacia un significado concreto procede de la orientación del signo indicador ( el gesto o la primera palabra) hacia un determinado objeto y, en última instancia, de la orientación afectiva hacia el objeto (Vygotski, L.; 1993: 87), por lo que la intencionalidad ligada a la actividad práctica y el lenguaje, al inicio independientes, se integran en el curso del desarrollo.

Según este autor, al comienzo son otras personas las que dan un primer sentido a la conducta o gesto del niño, quien relaciona su conducta primero con la situación general, luego con el sentido que le ha sido atribuido por otros en ese contexto situacional determinado, y sólo entonces su conducta comienza a tener ese sentido para él en ese contexto, modificándose la función que la conducta tenía al principio. Por ejemplo, el intento del niño de acercarse a un objeto llamativo se transforma en una indicación, en un medio de relación con otra persona, luego que los otros le han atribuido un significado y han actuado en consecuencia. Sólo después el niño da sentido a su gesto según lo que entendieron los otros, por lo que el niño es el último en dar un sentido a su conducta: "El gesto indicativo empieza a senalar por el movimiento lo que comprenden los demás; tan sólo más tarde se convierte en indicativo para el propio niño. Cabe decir, por lo tanto, que pasamosa ser nosotros mismos a través de otros; esta regla no se refiere únicamente a la personalidad en su conjunto sino a la historia de cada función aislada (...) Toda función psíquica superior pasa ineludiblemente por una etapa de desarrollo porque la función, al principio, es social. Este es el punto central de todo el problema de la conducta internay externa. (...) Toda función psíquica superior fue externa por haber sido social antes que interna; la fun- ción psíquica propiamente dicha era antes una relación social de dos personas. (...) Todas las formas fundamentales de comunicación verbal del adulto con el niño se convierten más tarde en funciones psíquicas. Podemos formular la ley genética general del desarrollo cultural del siguiente modo: toda función en el desarrollo cultural del niño aparece en escena dos veces, en dos planos; primero, en el plano social y después en el psicológico, al principio entre los hombres como categoria interpsíquica y luego en el interior del niño como categoría intrapsíquica" (Vygotski, L, 1995: 149-150). De este modo, y a diferencia de Piaget, para Vygotski el desarrollo no se orienta a la socialización, sino a convertir las relaciones sociales en funciones psíquicas.

En cuanto a la imitación, Vygotski sostiene que ésta no explica el lenguaje, pero la considera un proceso complejo que requiere también ser explicado y que presupone una determinada comprensión de la acción del otro. La imitación se encuentra también en animales no humanos, pero siempre requeriria una especie de entendimiento práctico. En relación a la actividad simbólica, Vygotski considera que ella tiene una función esencialmente organizadora que interviene en el proceso del uso de instrumentos y produce nuevas formas de comportamiento, permitiendo lo que él llama " el momento más significativo en el curso del desarrollo intelectual" que es cuando el lenguaje y la actividad práctica, dos lineas de desarrollo antes completamente independientes, convergen. Cuando el lenguaje hace su aparición junto con el empleo de los signos y se incorpora a cada acción, ésta se transforma y se organiza de modo nuevo. Así, para este autor, tanto en la filogenia como en la ontogenia el pensamiento y el lenguaje proceden de raíces genéticas independientes y tienen lineas de desarrollo distintas; sin embargo, existe una relación entre el pensamiento y el lenguaje que cambia durante el proceso de desarrollo, tanto en cantidad como en calidad, por lo que considera un error no tomar en cuenta esta relación en el estudio de los procesos cognitivos

En esta perspectiva, la utilización de signos por los niños no puede ser considerada una invención 
o algo transmitido por los adultos, sino más bien "algo" que surge de una historia de cambios o transformaciones cualitativas -aunque Vygotski no explica cuáles serían o cómo se darian-, a partir de lo que originariamente no es una operación con signos: "Cada una de esas transformaciones proporciona las condiciones necesarias para acceder al siguiente estadio, siendo a su vez condicionada por el estadio anterior; de este modo las transformaciones están vinculadas como estadios de un único proceso, de naturaleza histórica" (Vygotski; 1995:78). No se encuentran otras referencias respecto a cuáles serían estas transformaciones, pero es claro que se dan en la relación con otros y que en este proceso las actividades del niño adquieren un significado propio en un sistema de conducta social, de modo que ese significado depende de las formas concretas de interacción que el niño tenga en su medio.

Vygotski entiende la conducta del niño como propia de un sistema de actividad característico en cada etapa del desarrollo normal, que estaría determinado por dos factores: el grado de desarrollo orgánico y el grado de dominio en el uso de instrumentos. Sin embargo, admite al mismo tiempo cierta independencia de lo cultural y lo fisiológico al referirse al desarrollo del niño con retraso mental, sordera o ceguera, y a lo que él llama "las vías colaterales del desarrollo cultural", que serían los medios compensatorios ideados por la cultura como el sistema Braille o la lengua de señas. Estos medios compensatorios probarian que el desarrollo cultural de la conducta no está obligatoriamente relacionado con una u otra función orgánica y que sería independiente de uno u otro aparato fisiológico (Vygotski, L.; 1995),

\section{ElenfoquedeMaturana}

La postura de Maturana respecto a la intencionalidad del niño que aún no opera en el lenguaje se discutió ya más arriba. En cuanto a la referencia que el niño haría a objetos externos independientes de los participantes en la interacción, pre- vio a su operar en el lenguaje, Maturana piensa que eso no es posible y, como ya se ha dicho, su consideración relativa a que no se puede tener acceso a una realidad objetiva, independiente de lo que el observador hace, es un aspecto central en su teoria. Para el caso de la acción del niño pequeño en interacción con otros, es el observador de la conducta del niño quien la describe como conducta lingüistica porque ve cada elemento conductual como una palabra que señala un objeto o entidad existente, es decir, como si la conducta del niño se diera en el mismo dominio en que se da su propia conducta (la de quien es observador). Sin embargo, en el operar social del niño pequeño no hay objetos, pues él lo que hace es moverse en la coordinación conductual de la relación propia del grupo social al que pertenece. Los objetos sólo existen cuando el niño opera en el lenguaje, en la recursión de coordinaciones conductuales consensuales: "En el dominio social humano, y como resultado de las interacciones que tienen lugar entre los miembros de una sociedad humana, hay lenguaje cuando hay recursividad lingüística; es decir, cuando un observador ve coordinación conductual sobre la coordinación conductual. (...) De esto resultan, por una parte, la producción de un mundo de acciones y objetos que sólo tienen existencia y significado en el dominio social en que surgen, y por otra parte, la producción de la autoobservación, que nos lleva a distinguirnos como objetos a nosotros mismos y a nuestras circunstancias, en la reflexión que constituye la autoconciencia como fenómeno que también sólo tiene existencia y sentido en el dominio social. (Maturana, H. 1995: 12).

Al contrastar lo postulado por Vygotski y por Maturana, hay al menos dos aspectos en los cuales ambos autores parecen acercarse; el primero se refiere a lo que Vygotski llama el desarrollo social de los procesos cognitivos, en el sentido que este desarrollo sería dependiente de la cultura, de las condiciones concretas de vida de la persona. Vygotski sostiene que los procesos cognitivos, y por lo tanto el conocimiento y la comprensión del mundo, se desarrollan según las condiciones concretas y las formas de interacción social en que se viva, por lo 
que estos procesos cambiarían cuando las circunstancias histórico-sociales cambian. El segundo de estos aspectos se refiere a la descripción que Vygotski hace de la actividad pre-lingüistica no intencionada del niño, sosteniendo que en la generación del lenguaje se dan transformaciones cualitativas de "algo" que no es operaciones con signos pero que deriva en ellas en un proceso histórico en el cual surge el lenguaje y donde el sentido de la propia conducta del niño es atribuido primero por los otros, siendo sólo a partir de tal atribución que el niño otorga sentido a su conducta.

El primer aspecto mencionado que implica la modificabilidad de los procesos cognitivos, hace pensar en la concepción de aprendizaje como "deriva ontogénica" de Maturana, quien sostiene que el curso de esta deriva se configura en cada instante según la contingencia de sus interacciones con el medio (Maturana, H. 1982). El segundo aspecto que implica la recurrencia de interacciones y el entendimiento práctico en el proceso de generación del lenguaje, hace pensar en la explicación de Maturana respecto al desarrollo de las coordinaciones conductuales consensuales. Sin embargo, esta similitud es solo aparente, puesto que existe una contradicción en el punto de partida de ambas explicaciones: la concepción del fenómeno lingüístico. Para Vygotski, el pensamiento y el lenguaje son dos cosas distintas, independientes en su génesis y en su desarrollo, pero dependientes de un proceso evolutivo que los condiciona. El lenguaje es un instrumento del pensamiento que permite reflejar la realidad, y lo que define o caracteriza al simbolo (signo-palabra) es, precisamente, su referencia a lo objetivo. Para Maturana en cambio, el lenguaje es una dinámica relacional, no un instrumento; en el camino explicativo de la objetividad en paréntesis el lenguaje es una distinción que el observador hace del modo de fluir en las relaciones que se dan en las interacciones de dos o más personas, sólo cuando el lenguaje ocurre surge el observador que, como todo ser vivo, está determinado estructuralmente por lo que su propia dinámica estructural es la que define cuáles son las perturbaciones que acepta en cada momento, y no puede distinguir entre ilusión y percepción en la experiencia por lo que no puede hacer referencia a un mundo objetivo independiente de él, de sus habilidades como observador. Según la biología del conocimiento, tanto la filogénesis como la ontogénesis son procesos de deriva natural, sistémicosy epigenéticos, donde el curso que siguen los cambios del organismo se constituye momento a momento en el encuentro del sistema con sus circunstancias, sin que exista presión o fuerza de alguna naturaleza que les de una dirección o finalidad determinada (Maturana H.; 1989, 1990a; 1995)

\section{Conclusiones en el marcode la bio- logiadel conocimiento}

Las teorias de Piagety Vygotski sobre el lenguaje suponen un desarrollo separado e independiente, al principio, del pensamiento y del lenguaje, y este último es concebido como un instrumento del pensamiento que permite la comunicación. Para Piaget, el lenguaje se construye progresivamente a partir de la inteligencia individual como toda adquisición cognoscitiva, y su constitución permite recién el intercambio interindividual y el comienzo de la vida social del niño. Para Vygotski en cambio, el lenguaje se origina en la vida social, a partir de la cual se hace posible la reorganización de los procesos cognitivos del niño y su desarrollo intelectual. Si bien estas explicaciones sobre el surgimiento del lenguaje en el niño tienen diferencias importantes, en ambas el lenguaje es concebido como instrumento del pensamiento que sirve para comunicarse con otros sobre objetos que están en el mundo, y las primeras palabras o emisiones significativas del niño son entendidas como la simbolización de algo externo que existe con independencia de los participantes en la interacción y que preexiste a su operar en el lenguaje.

Para Piaget, laintencionalidad de los actos del niño pequeño es un elemento central en la imitación que prepara la adquisición del lenguaje articulado, socialmente transmitido por educación, y no considera la importancia del contexto situacional y de la relación afectiva en la interacción puesto que para él lo social 
sólo ocurre a partir de la adquisición del lenguaje. Piaget no explica el surgimiento del lenguaje porque no propone un mecanismo generativo para él, sino que lo engloba en las adquisiciones cognoscitivas que son posibles gracias a la inteligencia que es previa. En esta perspectiva, la actividad lingüistica se funda en supuestos explicativos de la actividad interna del niño -intenciones, pensamiento,etc.-, en relación con su actividad externa o conducta, la que seria resultado de la primera.

Vygotski, por su parte, descarta el elemento intencional en el niño pequeño y esta consideración comprueba para él la independencia de las líneas de desarrollo filogenético y ontogenético del lenguaje y del pensamiento. El autor reconoce que las primeras emisiones verbales inteligibles hacen referencia a toda una situación incluida la actividad del propio niño y propone que la utilización de signos deviene de algo que ocurre en las interacciones recurrentes del niño y el adulto que no es operación con signos, pero al sostener que el análisis genético del lenguaje debe explicar la transición desde el nivel en el que el niño utiliza la función indicativa hasta el nivel en que aparece la función simbólica, aborda esta cuestión principalmente desde la perspectiva del desarrollo del significado de las palabras o conceptos, (Wertsch, J. 1988), por lo que su análisis se centra principalmente en el lenguaje ya constituido y no en sus condiciones de generación (Ibáñez, N. 1999).

Para la biología del conocimiento de Humberto Maturana, el interés no está en la descripción del lenguaje como un fenómeno dado sino en explicar sus condiciones de generación: "La explicación cientifica del lenguaje como un fenómeno biológico consiste en la proposición de un mecanismo generativo que produzca las dinámicas de interacciones y coordinaciones de acciones que un observador distingue como lenguajear. Tal explicación debe mostrar como el lenguaje surge en las interacciones de los sistemas vivientes como sistemas determinados estructuralmente, y cómo constituye, en cuanto dominio de coordinaciones de acciones, un dominio fenoménico en el cual todo lo que hacemos en el lenguaje en la práctica del vivir puede ocurrir, y ocurre, cuando ciertas contingencias históricas ocurren" (Maturana, H. 1997:52). En esta perspectiva no hay separación entre lenguaje y pensamiento; no puede haberla, puesto que la única forma de conocer un mundo es generándolo en el lenguaje que es un modo de convivencia, una dinámica relacional. Para Piaget y Vygotski el lenguaje es un instrumento del pensamiento humano, para Maturana el observador surge en el lenguaje; el lenguaje es lo que nos hace humanosy, por lo tanto, en su visión sólo somos en el lenguaje.

Según Maturana, el lenguaje se funda en la emoción de aceptación del otro que es propia de nuestra biología y que él llama "amor". El amor es la emoción fundamental que hizo posible la historia evolutiva a la que pertenecemos; es decir, es la emoción que permitió el vivir en espacios de aceptación mutua donde se pudo dar la recurrencia de las interacciones, en el fluir de las cuales surgieron las coordinaciones conductuales consensuales y pudieron estas coordinaciones hacerse recursivas y determinar un modo de vivir que se conserva. Del mismo modo que debió ocurrir en el origen del lenguaje, sólo en el espacio relacional en el cual el amor tiene presencia se puede dar al comienzo, en el niño, la recurrencia de las interacciones que permite la expansión de las coordinaciones de acción y su transformación en coordinaciones de acciones consensuales, las que al ser recursivas constituyen lenguaje. (Maturana, H.; 1988; 1989; 1992a, 1995).

De este modo, en la perspectiva de la biología del conocimiento el mundo surge en el lenguaje, no preexiste a él. Cuando un objeto fisico, relacional o de cualquier índole se constituye para el niño, es el producto de una historia de coordinaciones de acciones que va configurando o construyendo ese objeto como tal para ese niño, según sea esa historia. Aquí, el lenguaje no es sólo la posibilidad que tenemos de hacer referencia a una realidad, es sobre todo lo que genera realidad e involucra mucho más que la actividad verbal. Los objetos (entidades) sólo surgen en el lenguaje, es decir, en el flujo del operar en coordinaciones de acciones consensuales recursivas. Antes del lenguaje no hay objetos, no hay simbolos, no hay intencionalidad. 


\section{Implicancias educativas}

Aceptar la concepción de lenguaje como coordinaciones de acciones consensuales de coordinaciones de acciones consensuales en la objetividad en paréntesis, implica hacerse cargo de que lo que se distingue tiene que ver con uno como observador y que, por lo tanto, todo lo distinguido es constituido en esa distinción porque no puede haber acceso a una realidad "objetiva". No se niega la existencia de una realidad, pero no existe un mecanismo mediante el cual se pueda tener acceso a ella como algo independiente de lo que somos-hacemos. El operar como observadores surge en el momento en que surge el lenguaje, el aprendizaje del mundo se configura con las distinciones que hacemos en el lenguaje en interacción con otros en un medio particular, en lo que Maturana llama una co-deriva, en la cual los cambios estructurales de las personas que interactúan recurrentemente siguen un curso contingente a esas interacciones, por lo que nuestro mundo puede cambiar al cambiar el curso de nuestras interaccciones. Esto es siempre posible y constituye un aspecto particularmente importante en la proyección pedagógica de esta visión del lenguaje.

En nuestra cultura "objetiva", el interés de los padres y maestros respecto al aprendizaje del lenguaje de los niños se focaliza en que ellos aprendan las palabras que designan los objetos (entidades) y sus características o propiedades, sin mayor preocupación por el modo en que ese objeto será construido porque se parte del supuesto que ese objeto está allicon independencia del observador. En esta nueva mirada, el interés se traslada a las coordinaciones de acciones recurrentes cuyo desarrollo permitirá esa construcción. Es decir, se traslada al modo en que se va dando la historia de las coordinaciones de acciones, que es desde dónde surgirá el objeto (físico o relacional) cuando haya recursividad en las coordinaciones de acciones consensuales en las que el niño o niña participa con uno. Así, la silla surgirá como objeto "silla" particular según haya sido la historia de interacciones recurrentes que ese niño o niña tuvo en relación con lo que puede estar implicado en las coordinaciones de acciones consensuales donde la "silla" surge. Por ejemplo, sentarse o subirse; donde el sentarse o el subirse no está separado de todo lo que le da sentido, lo que tiene presencia en esas coordinaciones de acciones consensuales y que Maturana llama "coherencias operacionales", como sería el espacio de movimiento del niño, la corporalidad de los participantes en la interacción, el espacio emocional en que esta interacción ocurre, la actitud de la madre, lo que pasa antes y después, el agrado (o desagrado) que se sienta, etc., etc.. En este ejemplo, parece simple concluir que no debe haber diferencias importantes en cómo se constituye el objeto "silla" y su ámbito de coherencias operacionales para los niños de culturas similares, pero lo interesante es hacerse cargo que del mismo modo surgirá para el niño o niña el "yo y el otro", lo "mío y lo tuyo", lo "bueno y lo malo", lo "deseable y lo indeseable", lo "inteligente y lo tonto", lo "hermoso y lo feo", y ... todo el resto del mundo.

La existencia depende de lo que el observador hace, el observador trae a la mano lo que distingue: "... la unidad distinguida existe en el acto de traerla a la mano, pero existe en la contrapartida de un ámbito de coherencias en la cual tiene sentido y que se trae a la mano al hacer la distinción de la unidad" (Maturana, H.; 1992a:34). En esta concepción del lenguaje, las palabras son distinciones de coordinaciones de acciones consensuales en el flujo de coordinaciones de acciones consensuales, por lo que las palabras no revelan el objeto sino ocultan las coordinaciones deaccionesquedistinguen.

Padres y profesores sabemos que, de algún modo, nuestras interacciones recurrentes con los niños y niñas configuran el sentido del mundo en que viven, pero al creer que este mundo preexiste a su distinción nos esforzamos en dar la designación exacta o la explicación correcta de las cosas "como son". Al entender ahora que esto no es así, necesitamos hacernos cargo de que la generación de ese mundo depende de nuestro actuar, del flujo de las coordinaciones de acciones consensuales en las que operamos en nuestro convivir con los niños y niñas, y que un cambio en el curso que sigue ese flujo posibilita siempre un cambio en las distinciones que ellos y nosotros podamos hacer. 


\section{Bibliografia}

\section{Bruner,J erome}

1989 Acción, pensamiento y lenguaje . Alianza Editorial: Madrid 1989.

\section{Ibáñez, $\mathbf{N}$.}

1998 "Aportes a la psicopedagogia, a partir de una nueva concepción del lenguaje". Rev. de Tecnologia Educativa. Vol XIII No 1 pp.69-81. Santiago.

1999 "El surgimiento del lenguaje en el niño" Tesis Doctoral. Universidad Academia de Humanismo Cristiano-PIIE. Santiago.

\section{Luriay Yudovich}

1979 Lenguaje y desarrollo intelectual. Pablo del Rio; Madrid.

\section{Maturana, $\mathrm{H}$.}

1982 "Reflexiones: aprendizaje o deriva ontogénica". Arch, Biol. Med. Exp. Vol. 15: 261.271 1988 "Reality: The search for objetivity or the quest for a compelling argument" Irish Journal os Psychology: vol. 9 (1): 25-82

1988 Ontología del conversar. Rev. de Terapia Psicológica Año VII N ${ }^{\circ} 10$. Santiago.

1989 “Dónde está la mente?”. Transcripción conferencia Universidad Técnica FSM . $8^{\circ}$ Congreso de Psicologia Clínica. Valparaíso.

1990 Emociones ylenguaje en educación y politica. Hachette. Santiago.

1990 Biología de la cognición yepistemología.Ed.Universidad La Frontera. Temuco.

1991 Transcripciones de entrevistas personales sobre el lenguaje a N. Ibáñez, F.Farias y V. Lobos. Universidad Matropolitana de Ciencias de la Educación. Santiago.

1992 El sentido de lo humano. Hachette. Santiago.

1993 De la biología a la psicología Ed. Synthesis, Viña del Mar.

1997 La objetividad. Un argumento para obligar Ediciones Dolmen, Santiago.
Maturana, H. y Varela, F.

1984 El árbol del conocimiento. Edit. Universitaria. Santiago, Chile. $2^{a}$ edición. 1995 De máquinas y seres vivos. Universitaria. Santiago. $3^{\mathrm{a}}$ ediciōn.

\section{Maturana H. y Ludewing, $\mathrm{K}$.}

1992 Conversaciones con Humberto Maturana: preguntas del psicoterapeuta al biólogo. Edic. Universidad La Frontera, Temuco.

\section{Maturana, H. y Verden-Zöller, G.}

1993 Amoryjuego. Fundamentosolvidados delo humano. Edit. Instituto de Terapia Cognitiva. Santiago, Chile. $3^{\mathrm{a}}$ edición.

\section{Piagt, J ean}

1980 Problemas de psicología genética. Ariel; Barcelona. $4^{\mathrm{a}}$ edición.

1981 Biología y Conocimiento. Siglo Veintiuno; Barcelona. $6^{\mathrm{a}}$ edición

1982 La formación del símbolo en el niño. Fondo de Cultura Económica; México. $7^{\text {a }}$ edición.

1983 Seis estudios de psicología. Ariel; Barcelona.

\section{Piaget, J . elnhelder B.}

1982 Génesis de las estructuras lógicas elementales. Edit. Guadalupe; Buenos Aires. $4^{\mathrm{a}}$ edición.

\section{Piaget, J . y Chomsky, N.}

1983 Teorias dellenguaje-teorias del aprendizaje.El debate entre J. Piagety N. Chomsky. Critica; Barcelona.

\section{Vygotski, L.S.}

1988 Pensamiento y Lenguaje. Comentarios criticos de Jean Piaget. La Pléyade; Buenos Aires

1995 ElDesarrollo delos procesospsicológicos superiores. Crítica; Barcelona. $3^{\mathrm{a}}$ edición.

1993 Obras escogidas II. Visor; Madrid.

1995 Obras Escogidas III. Visor; Madrid.

\section{Wertsch,J ames}

1988 Vygotskiy la formación social de la mente. Paidós; Buenos Aires. 